\title{
Throughput-Range Tradeoff of Wireless Mesh Backhaul Networks
}

\author{
Harish Viswanathan, Senior Member, IEEE, and Sayandev Mukherjee, Senior Member, IEEE
}

\begin{abstract}
Wireless backhaul communication is expected to play a significant role in providing the necessary backhaul resources for future high-rate wireless networks. Mesh networking, in which information is routed from source to destination over multiple wireless links, has potential advantages over traditional single-hop networking, especially for backhaul communication. We develop a linear programming framework for determining optimum routing and scheduling of flows that maximizes throughput in a wireless mesh network and accounts for the effect of interference and variable-rate transmission. We then apply this framework to examine the throughput and range capabilities for providing wireless backhaul to a hexagonal grid of base stations, for both single-hop and multihop transmissions for various network scenarios. We then discuss the application of mesh networking for load balancing of wired backhaul traffic under unequal access traffic conditions. Numerical results show a significant benefit for mesh networking under unbalanced loading.
\end{abstract}

Index Terms-Load balancing (LB), mesh networking, multihop, routing, scheduling, WiMax.

\section{INTRODUCTION}

W IDE-AREA wireless broadband access is poised to become ubiquitous with the advent of technologies such as WiFi (802.11) [1], WiMax (802.16 family of standards) [2] and high data rate cellular systems [3], [4]. However, the inverse relationship between data rate and base station (BS) range, together with limitations on range imposed by transmit power, results in deployments with large numbers of BSs to cover a given area. An important factor that will determine the success or failure of such an approach will be the availability of inexpensive backhaul bandwidth to a large number of BSs. Wireless backhaul (e.g., IEEE 802.11s [5] and 802.16d [6]) is expected to play a significant role in providing the necessary backhaul solution for such networks. A common characteristic of such wireless backhaul systems is the use of mesh or multihop networking to improve efficiency.

Mesh networking, in which information is routed from source to destination over multiple wireless links, has potential advantages over traditional single-hop networking, such as spatial reuse for increased capacity, coverage enhancement [7]-[11], load balancing (LB) through route diversity [12], and user cooperation diversity, and has been studied extensively in the context of wireless ad hoc networks.

In this paper, we examine wireless mesh networking from the perspective of providing wired network connectivity to a number of wireless BSs that provide wireless access to end users. The

Manuscript received January 12, 2005; revised May 10, 2005

The authors are with the Bell Laboratories, Lucent Technologies, Murray Hill, NJ 07974 USA (e-mail: harishv@lucent.com; sayan@lucent.com).

Digital Object Identifier 10.1109/JSAC.2005.862408 fixed nature of the network and throughput requirements permit us to consider centralized routing and scheduling schemes to maximize the throughput and range. The routing problem deals with the question of how different information flows for different destination nodes in the network are routed from the wired BS. The scheduling problem relates to determining the duration for which each transmission scenario, i.e., a given set of transmitter-receiver pairs that are simultaneously communicating, should be active. We first show that, given the set of all possible transmission scenarios, a linear programming (LP) optimization solves the joint routing and scheduling problem. We then apply the LP to study the benefit of multihop technology for providing backhaul in a network of BSs placed on a hexagonal grid, by evaluating the throughput-range tradeoff achieved by single-hop and multiple-hop methods for a variety of antenna heights, transmit powers, beamforming gains, and site-to-site distances. Our results indicate that, for the propagation model considered, locating the wired BS transmitter on a tall tower and transmitting at high power on a single-hop provides throughput comparable to that achieved by multihop technology with smaller transmit powers and lower antenna heights.

Current high spectral efficiency systems such as third-generation cellular systems based on code-division multiple access (CDMA) employ universal frequency reuse and are usually interference limited [13]. A key feature of our analysis is that instead of assuming only a single transmission within a given radius of a receiver, as is usually done in the literature, we explicitly take into account the effect of interference at the receiver due to other transmitters. Further, we assume variable-rate transmissions that are matched to the available signal-to-interference and noise ratio (SINR) and implemented through adaptive coding and modulation, as is the case with packet-data standards [1]-[4], as opposed to employing power control to achieve a given target SINR (which can be less spectrally efficient).

Our formulation of an LP to jointly optimize routing and scheduling within the framework of variable-rate transmissions dependent on the interference power is in contrast to that of other authors, who either do not consider the joint routing-scheduling problem [16], or focus on power control in place of variable-rate transmission [14], or neglect the effect of interference on transmission rates [15], [17], or do not formulate the routing problem as an LP [10]. In addition, in this paper, we also provide a systematic evaluation of the throughput-range tradeoff for backhaul communication.

We also consider the benefit from LB that is made possible by wireless multihop. In this case, every BS is connected to the wired network through a wireline connection. However, the capacity of the wireline connection is less than the access traffic 
generated at some of the BSs. These BSs can divert traffic to other BSs using the wireless mesh backhaul thereby increasing the amount of access traffic that can be carried to the wired network. We show that the LB problem can also be treated within the LP framework. We then provide a numerical evaluation of the throughput gain that can be achieved through LB.

The paper is organized as follows. We describe our framework for solving the routing and scheduling problems and a heuristic algorithm for selecting transmission scenarios in Section II. In Section III, we describe the evaluation of throughput-range tradeoff for a regular network consisting of a hexagonal grid of BSs and highlight the key results. In Section IV, we describe the LB problem and present results that show the extent of benefit that LB can provide. We conclude with a summary and directions for future work in Section V.

\section{Wireless NetWork Flow PROBlem}

\section{A. System Model}

1) Locations of Nodes: The model we study is that of a network with multiple nodes. Each of these nodes represents a BS serving subscribers in a given region, which we shall call a cell in analogy to conventional cellular wireless systems. Some of these BSs, called access points (APs), are assumed to have a wired connection to the backhaul network. The remaining BSs correspond to so-called extension points (EPs) in the sense of extending the range of the wired BSs (APs) over a wider area.

All APs are assumed identical, and all EPs are also assumed identical.

2) Channel Model: While our analysis is applicable to any model of path loss between a transmitter and receiver, for concreteness, we assume the Erceg-Greenstein model. Since the EPs and APs are all stationary, there is no fast fading on the links. Thus, the maximum rate that can be supported on a given wireless link is a function only of the transmit power, distance between transmitter and receiver, shadow fading on the link, and interference power at the receiver from neighboring node transmissions. As we shall see, the analysis does not require assumptions on whether transmit power control is employed, or on the shadow fading model. However, in the simulations, we have set the transmit power to be equal at all EPs and at all APs, but different between EPs and APs. The shadow fading model is the customary log-normal model.

3) Traffic Model: In the present section, we focus on the downlink, i.e., the transfer of information from the APs to the EPs, and assume for simplicity that all traffic for all subscribers in the cell arrives at only one AP. Note that throughout this paper, we are only interested in the aggregate traffic per cell and, thus, the locations of the individual subscribers in each cell are irrelevant to our analysis, so long as we make the assumption that the aggregate traffic demand per cell is constant over time. Thus, when we write "traffic intended for an EP," we mean "traffic intended for all subscribers served by that EP."

Note that in practical systems, transmission intervals are usually divided into time slots, and information is transmitted in the form of packets. However, if the time slot duration is sufficiently small, and a transmission interval can comprise multiple time slots, then the duration of a transmission interval is effectively arbitrary, and we can employ the above approximation of

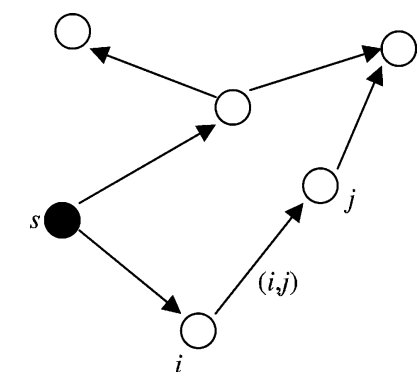

Fig. 1. An example of a network with six EPs, one AP (marked $s$ ), and several links, represented by arrows. An example link $(i, j)$ between nodes $i$ and $j$ is also shown.

focusing only on individual bits of the packets, rather than the packets themselves.

\section{B. Statement of the Wireless Network Flow Problem}

All traffic intended for a particular destination EP arrives in a continuous stream of bits at a specific AP and is transmitted to other EPs en route to the destination EP. The bits intended for the destination EP reside in buffers at the intermediate EPs on the path between the AP and the destination EP. These buffers are assumed to be of infinite length, so that bits are never lost due to queues overflowing. We assume that the buffers in the intermediate nodes are never empty since the focus is on the average throughput achievable and by appropriate scheduling of transmissions, buffers can be maintained to be nonempty just before transmission. This makes the movement of data through the network analogous to the flow of fluid through a network of pipes. By analogy to the fluid flow problem, the portion of the total number of bits that travels through a given link (edge of the graph) is called the flow on that edge. The traffic intended for each EP is called a commodity and indexed by the label of the EP. The throughput to each destination EP is simply the ratio of the total number of bits it receives in a certain time interval to the total transmit time allocated for these bits at the AP and at the various intermediate EPs.

Suppose that the network has $n$ EPs, each EP $d$ having the same demand $f$, which represents the number of bits we would like to be able to receive at this EP from the source (AP). We model the network itself as a directed graph, where an edge between nodes $i$ and $j$, denoted $(i, j)$, represents a wireless link from node $i$ to node $j$. The flow corresponding to a given commodity $d$ (i.e., traffic intended for EP $d$ ) through the edge $(i, j)$ is denoted $x_{i j}^{d}$ [21, p. 369]. Thus, $x_{i j}^{d}$ is the portion of the total number of bits between $s$ and $d$ that flows through the edge $(i, j)$. Also, each link $(i, j)$ has a finite capacity $R_{i j}$, which represents the maximum rate (in bits/s) of transmission on that link, such that the transmitted bits are received with arbitrarily small probability of error at the receiver.

However, the capacity on each link depends upon the set of other simultaneously transmitting links because of interference. Thus, we have to consider all possible situations in which only a subset of the total set of possible links can be active at any given time. For brevity, we shall refer to each subset of simultaneously active links as a transmission scenario. For example, Fig. 1 shows a network with six EPs, one AP (marked $s$ ), and several links represented by arrows between nodes, of which 


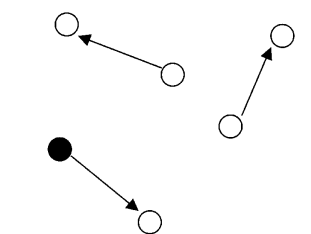

Transmission scenario 1

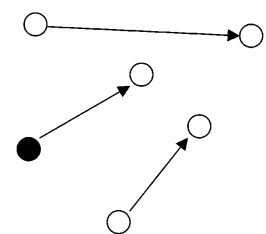

Transmission scenario 2
Fig. 2. Two examples of transmission scenarios for the network and links shown in Fig. 1. Each transmission scenario is a set of links that can be simultaneously active. In this particular example, the links in the two transmission scenarios are disjoint, but this is not required.

an example $(i, j)$ is also shown. In Fig. 2, we show two possible transmission scenarios for the network and links in Fig. 1. In this example, the links in the two transmission scenarios are disjoint, but this is not true in general.

Suppose that we have a set of $N$ possible transmission scenarios, labeled $\mathcal{L}_{1}, \ldots, \mathcal{L}_{N}$. Now, in order to satisfy the demand for each commodity, i.e., for the traffic intended for each destination EP, we not only have to choose the paths and flows for each transmission scenario, but also the allocation of the total flow (over all transmission scenarios) to each transmission scenario. The flow corresponding to destination EP $d$ through link $(i, j) \in \mathcal{L}_{t}$, i.e., through the link $(i, j)$ in transmission scenario $t$, is denoted by $x_{i j}^{d}(t)$. If the transmission time allocated to the links in transmission scenario $t$ is $T_{t}$, then the total transmission time over all $N$ transmission scenarios is $\sum_{t=1}^{N} T_{t}$.

The objective is to minimize the total transmission time required for all EPs to receive their demanded number of bits $f$, i.e.,

$$
\min \sum_{t=1}^{N} T_{t}
$$

subject to each EP receiving its demand $f$ over the total of $N$ transmission scenarios

$$
(\forall d, j \neq d) \sum_{t=1}^{N} \sum_{i} x_{i j}^{d}(t)-\sum_{t=1}^{N} \sum_{k} x_{j k}^{d}(t)=0
$$

and

$$
\sum_{t=1}^{N} \sum_{i} x_{s i}^{d}(t)=\sum_{t=1}^{N} \sum_{k} x_{k d}^{d}(t)=f
$$

(which is the condition representing the requirement that these bits are generated at $s$ and intended for $d$ ), and to the usual capacity constraints on each link in each transmission scenario

$$
\forall t \text { and } \forall(i, j) \in \mathcal{L}_{t}, \quad \sum_{d} x_{i j}^{d}(t) \leq R_{i j}(t) T_{t}
$$

where now we account for the dependence of link capacity on the transmission scenario by denoting the capacity of link $(i, j)$ in transmission scenario $\mathcal{L}_{t}$ by $R_{i j}\left(\mathcal{L}_{t}\right)$, or $R_{i j}(t)$ for simplicity. Under the assumption of time-invariant channel conditions, this is given by

$$
R_{i j}(t)=\log _{2}\left(1+\eta \frac{\frac{P \Gamma_{i j}}{d_{i j}^{\gamma}}}{1+\sum_{\substack{(k, l) \in \mathcal{L}_{t}: \\(k, l) \neq(i, j)}} \frac{P \Gamma_{k l}}{d_{k l}^{\gamma}}}\right)
$$

where $P$ is the transmit power, $d_{i j}$ and $\Gamma_{i j}$ are, respectively, the distance and shadow fading between nodes $i$ and $j$, and $\gamma$ is the path loss exponent. ${ }^{1}$ The factor $\eta$ represents any implementation margin relative to the rate given by the Shannon formula [18]. Note that achieving this rate requires variable-rate transmission that is matched to the received SINR, which can be implemented in practice through adaptive coding and modulation.

The common throughput to each of the EPs is, therefore, given by

$$
\frac{f}{\sum_{t=1}^{N} T_{t}}
$$

Remark: Note that the LP problem formulation in (1-a)-(1-c), if solved exactly over the entire space of all possible transmission scenarios, yields an exact value for the throughput. In fact, this value is an upper limit on the achievable throughput in a practical system. This is due to several reasons, among them the following:

1) The interference due to far-away transmitters is not exactly zero;

2) The Shannon formula (2) is an upper limit on the rate achievable for a given SINR.

However, there is a more immediate practical issue with obtaining a value for the throughput from the LP problem (1-a)-(1-c), namely, that the set of all transmission scenarios and, thus, the size $N$ of the LP problem, may be too large for the LP problem to be solved exactly. We envisage an application of the wireless network flow problem to the design and installation of backhaul networks, where the topology of the network is invariant and the channels and traffic load are quasi-static. In these situations, even if the LP problem (1-a)-(1-c) is of large complexity, it may be possible to solve it to determine static routing schemes that can then be signaled to the different BSs. However, the solution of the "full" (i.e., over all transmission scenarios) LP problem may be impossible even for modest numbers of EPs: if there are $n$ EPs and one AP in the network, there are a maximum of $L=\left(\begin{array}{c}n+1 \\ 2\end{array}\right)$ possible links and, thus, a total of $N=2^{L}-1$ nonempty transmission scenarios, which is very large even for moderate values of $n$. Thus, for most practical problems, it is impossible to enumerate the set of all scenarios, and the LP problem (1-a)-(1-c) must be solved over a reduced set of transmission scenarios. We provide an overview of the heuristics to do so in Section II-C, and propose an algorithm to enumerate a tractably large subset of transmission scenarios. It must be noted that solving the LP problem over a reduced set of transmission scenarios yields only a lower bound on the maximum throughput attainable (which is obtained by solving the full LP problem). The particular combination of storage and computing capabilities available to attack the LP problem determines the maximum tractable size of the set of transmission scenarios and, therefore, the closeness of the throughput value obtained from the solution of the reduced problem to the maximum throughput.

\footnotetext{
${ }^{1}$ The path loss intercept and additive Gaussian noise power can be absorbed into the transmit power $P$ and, hence, both are set equal to 1 .
} 


\section{Greedy Algorithm for Selection of Transmission Scenarios}

We assume that all transmissions are point-to-point (so each transmitter transmits to exactly one receiver, and each receiver receives from exactly one transmitter), and that no transmitting node can receive simultaneously. Thus, if any two links $(i, j)$ and $(k, l)$ are active simultaneously, we must have $i, j, k, l$ all distinct. These criteria reduce the size of the set of feasible transmission scenarios. Some authors [15] further reduce the size of this set by requiring that, e.g., no neighbor of a receiver can transmit (other than a chosen transmitter), or that all neighbors of both a chosen transmitter and a chosen receiver can neither transmit nor receive.

We propose a greedy algorithm for selection of transmission scenarios which can then be used within the LP framework to determine the maximum throughput. The algorithm is motivated by the fact that a good transmission scenario should consist of as many simultaneous transmissions as possible, while keeping the loss in SINR for each transmission due to interference from other transmissions small. A similar greedy algorithm with a similar objective has been proposed for uplink scheduling in CDMA systems [19].

The algorithm proceeds in steps with a single link added in each step to form a single transmission scenario. The algorithm is repeated $N$ times, each time with a randomly selected link from the set of allowed links as the first link in the transmission scenario, to form $N$ different transmission scenarios. Denote by $\mathcal{X}_{t}$ the set of links available from which a link can be chosen to add to transmission scenario $\mathcal{L}_{t}, t=1, \ldots, N$. Initially, $\mathcal{X}_{1}$ is the complete set of allowed links. The algorithm picks the first link in the transmission scenario at random and, subsequently, picks one link at a time, always choosing the link that maximizes the total transmission rate in the transmission scenario in a greedy fashion. If the total transmission rate can no longer be increased, then the algorithm is terminated with the current transmission scenario being chosen as $\mathcal{L}_{t}$. In order to avoid transmission scenarios that call for multiple transmissions from or receptions at a single node, each step which results in a new link $\ell^{*}$ being added to the transmission scenario $\mathcal{L}_{t}$ is followed by updating the set of available links $\mathcal{X}_{t}$ to eliminate those links that share a common node with the source or destination node of the most recently added link $\ell^{*}$. To pick multiple transmission scenarios $\mathcal{L}_{1}, \ldots, \mathcal{L}_{N}$ that together will ensure that data can be transmitted from any source node to any destination node, the final set of allowed links $\mathcal{X}_{t}$ is used as the initial set of allowed links $\mathcal{X}_{t+1}$ for the next transmission scenario selection. The goal is to ensure that each node appears on at least one path from the AP and, thus, no node is starved. After selecting a number of transmission scenarios $\mathcal{L}_{1}, \ldots, \mathcal{L}_{t}$, when the set of allowed links $\mathcal{X}_{t+1}$ becomes small, say having fewer members than some chosen value $k_{\text {min }}$, it is reset to include the set of all allowed links for selection of subsequent transmission scenarios $\mathcal{L}_{t+1}, \ldots, \mathcal{L}_{N}$. The algorithm is described formally as follows.

Recall that each transmission scenario $\mathcal{L}$ consists of a set of links each of which is identified by a transmitting node and a receiving node. Denote by $\ell$ an arbitrary link of a transmission scenario $\mathcal{L}$. The source and destination of $\ell$ are denoted $s(\ell)$ and

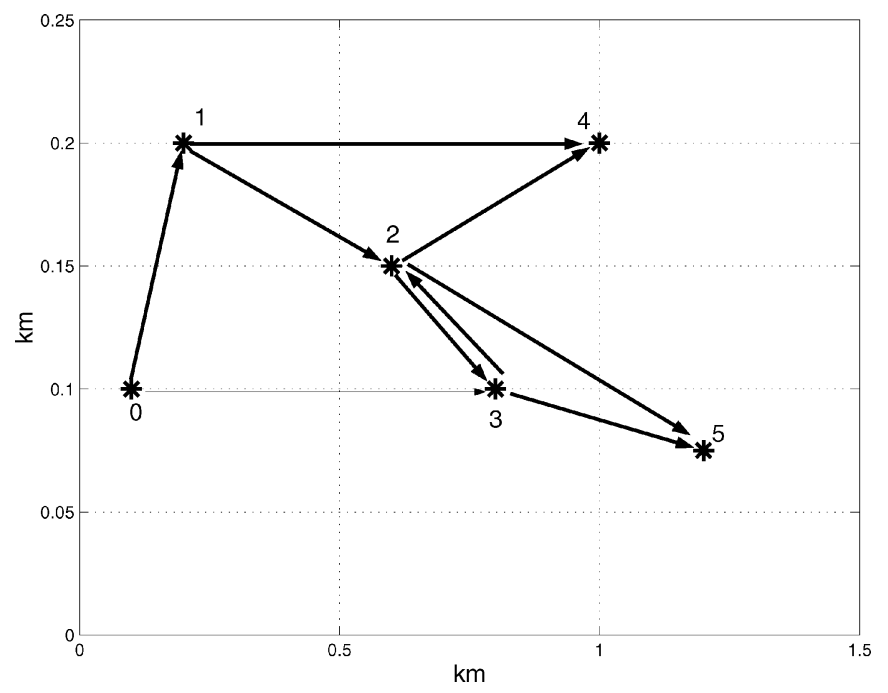

Fig. 3. Network with five randomly placed nodes, and nine links, marked as shown.

$d(\ell)$, respectively. Denote the total transmission rate achieved in the transmission scenario $\mathcal{L}$ by $S(\mathcal{L})$, which is given by

$$
S(\mathcal{L})=\sum_{\ell \in \mathcal{L}} R_{s(\ell), d(\ell)}(\mathcal{L})
$$

where $R_{i, j}(\mathcal{L})$ is as defined in $(2)$.

For $t=1, \ldots, N$

- If $t=1$ or $\left|\mathcal{X}_{t}\right|<k_{\min }$, set $\mathcal{X}_{t}=\{\ell$ : $(s(\ell), d(\ell))$ is an allowed link $\}$.

- Set $\mathcal{L}_{t}=\emptyset$.

- Set $\ell^{*}=$ a single link chosen at random from $\mathcal{X}_{t}$.

- while $S\left(\mathcal{L}_{t} \cup\left\{\ell^{*}\right\}\right)>S\left(\mathcal{L}_{t}\right)$,

- Set $\mathcal{L}_{t}=\mathcal{L}_{t} \cup\left\{\ell^{*}\right\}$

- Set $\mathcal{X}_{t}=\mathcal{X}_{t} \backslash \bigcup_{\ell \in \mathcal{X}_{t}}\{\ell:\{s(\ell), d(\ell)\} \cap$ $\left.\left\{s\left(\ell^{*}\right), d\left(\ell^{*}\right)\right\} \neq \emptyset\right\}$.

- Compute $\ell^{*}=\arg \max _{\ell \in \mathcal{X}_{t} \backslash \mathcal{L}_{t}} S\left(\mathcal{L}_{t} \cup\{\ell\}\right)$.

- Set $\mathcal{X}_{t+1}=\mathcal{X}_{t}$.

The throughput performance of the greedy algorithm is compared with that of brute force enumeration of all transmission scenarios for a five-node network $(n=5)$ with nine possible links illustrated in Fig. 3. For this network, there are a total of $N=26$ transmission scenarios that are possible.

We assume a bandwidth of $10 \mathrm{MHz}$ and log-normal shadow fading with a standard deviation of $8 \mathrm{~dB}$. The channel propagation model is taken to be the Erceg-Greenstein model [20] for an "intermediate" terrain (neither hilly nor flat, with moderate foliage), and described by the following parameters: reference distance $100 \mathrm{~m}, A=4, B=0.0065$, and $C=17.1$. All nodes transmit with $30 \mathrm{dBm}$ power, and all antennas are $10 \mathrm{~m}$ high.

We simulated the algorithm for multiple instantiations of the network shown in Fig. 3, each time with a different set of random shadow fades on the links. Fig. 4 shows the cumulative distribution function (CDF) of the throughput that is achieved with all 26 transmission scenarios compared with that achieved by 20,15 , and 10 transmission scenarios chosen according to the greedy algorithm. As seen from the figure, with about 


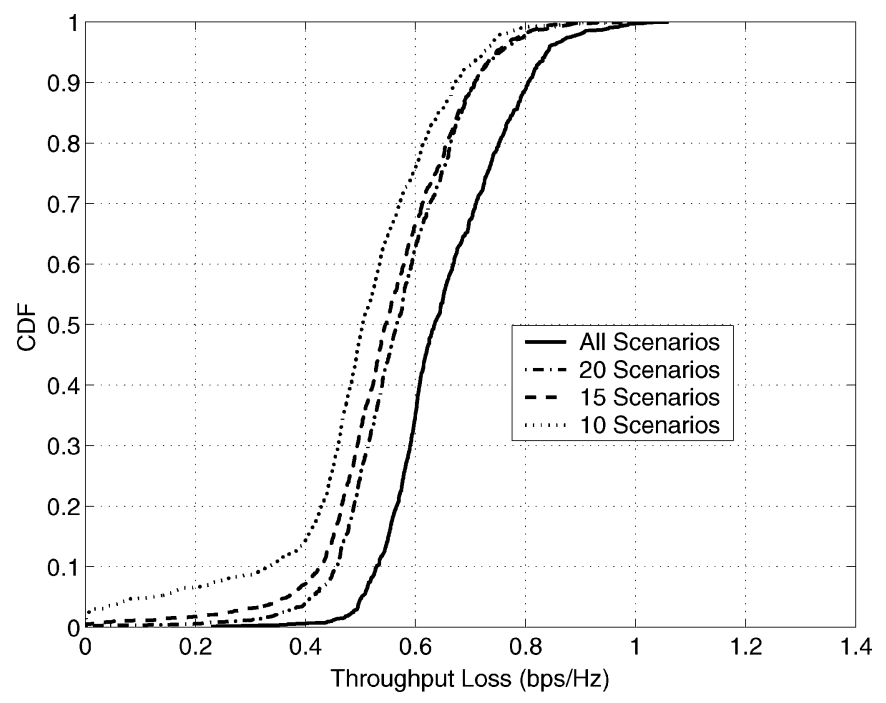

Fig. 4. Throughput as computed via the wireless network flow LP problem (1-a)-(1-c) over all 26 possible transmission scenarios, compared with that obtained over only 10,15 , or 20 transmission scenarios obtained from the greedy algorithm described in Section II-C.

$50 \%$ of the transmission scenarios, a significant fraction of the throughput is achieved for various link rates.

\section{Throughrut Range TradeOFF IN Regular Network}

\section{A. Network Model for Evaluation}

In this section, we compare the throughput per cell (i.e., per EP) versus the distance of the EPs from the AP for the special geometry where the AP is at the center of several concentric rings of EPs in such a way that the locations of the AP and the EPs fall on a hexagonal lattice. In other words, if we draw a hexagonal grid on the plane, the AP is at the center of one of the hexagons, and the EPs are at the centers of several "rings" of hexagonal cells surrounding the central cell containing the AP, as shown in Fig. 5 for the case of three rings.

This models the situation where, in order to save on infrastructure expenses of laying cable or fiber to each BS in a network, we try to extend the range of a given BS (the AP) with wired backhaul connection by using several other BSs (i.e., the EPs) that have no wired backhaul connection and can only communicate with the backhaul via the original BS. Each EP serves all users that lie within the region given by the hexagonal cell with this EP at its center. Thus, the throughput from the AP to the EPs must be large enough to support the user traffic requirements at the EPs.

We now focus on the special case where the throughput requirement to each EP is the same. Our goal is to fix the transmit powers of the AP and the EPs, and compare and contrast the extension in range possible with multihop routing versus single-hop routing, where the AP transmits in turn to each of the EPs, and no EP transmits to any other EP.

In the multihop case, we further require that links can only be formed between the AP and EPs of the first ring around the AP, or between EPs in adjacent rings, but an EP cannot transmit to any EP that is more than one ring away. With uniform spatially homogeneous traffic in all these cells, the throughput per EP only depends on the distance of the EP from the AP, i.e., on the

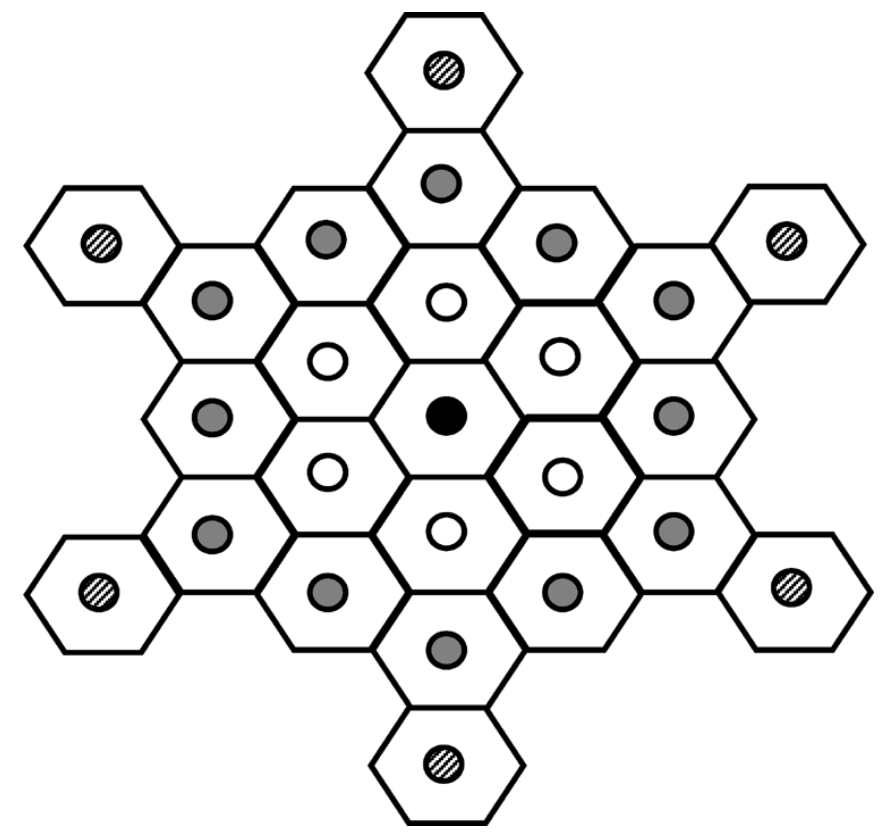

Fig. 5. A "regular" network with the BSs in the centers of hexagonal cells, for which the throughput versus range tradeoff is investigated. The AP is in the center, surrounded by EPs in three "rings." The throughput is obtained by solving the wireless network flow LP problem (1-a)-(1-c). We have restricted only 6 out of the 18 cells in the third ring to have EPs because otherwise the total number of scenarios becomes too large for the numerical LP solver to handle.

"ring" in which the EP is located. It is intuitively obvious that since the traffic for an EP in a distant ring needs to be routed through all of the intermediate rings of EPs, the throughput per EP decreases with the number of rings. The maximum number of rings of EPs that can be supported by an AP such that the throughput requirement is met at each EP is a measure of the range extension of the AP that is possible by using the EPs.

\section{B. Enumerating the Scenarios for the Multihop Optimization}

As before, we require that in each transmission scenario, every active transmitter has exactly one receiver corresponding to it, and every node that is part of a link in a transmission scenario is either a transmitter or a receiver but not both. Further, as discussed above, every transmitter-receiver pair must have either: 1) the AP as the transmitter, and an EP in the first ring as the receiver or 2) the transmitter EP and receiver EP located in adjacent rings, with the receiver EP in the "outer" ring and in a cell adjacent to the cell containing the transmitter EP. In other words, with reference to the example in Fig. 5, any link is either between the AP and a first-ring EP, or between a first-ring and a second-ring EP in adjacent cells, or between a second-ring EP and a third-ring EP in adjacent cells. We also focus on maximal transmission scenarios only, i.e., subsets of simultaneously active links such that no new links can be added to that subset, while still obeying the other restrictions concerning the subset (such as those listed above).

The greedy algorithm described in Section II-C could be applied to enumerate the transmission scenarios for this case as well. However, the greedy algorithm did not use any special properties about the structure of the network. In this case, the network has a high degree of spatial symmetry. This implies that if the LP given by (1-a)-(1-c) were to be solved over the 
complete set of scenarios, we would expect the scenarios with nonzero time allocations to be symmetric. We, therefore, describe here how to enumerate a set of symmetric transmission scenarios.

Note that the AP and EPs could have antennas with steerable beams. We study two extreme cases of these antennas, one where the antennas are omnidirectional, and one where the antennas have such narrow beams that there is no interference to any neighboring cell.

1) Narrow-Beam Antennas: In the narrow-beam case, the interference caused by any active link on any other link is assumed negligible and, thus, there are no more restrictions on the links in a transmission scenario than that $\ell_{1}$ and $\ell_{2}$ are links in the same scenario if and only if $\left\{s\left(\ell_{1}\right), d\left(\ell_{1}\right)\right\} \cap\left\{s\left(\ell_{2}\right), d\left(\ell_{2}\right)\right\}=\emptyset$. The maximal transmission scenarios for the given geometry are symmetric and can be enumerated by inspection for the case of two and three rings.

2) Omnidirectional Antennas: To enumerate the set of all feasible transmission scenarios in this situation, we also require that if a particular transmitter-receiver pair is included in a transmission scenario, then no neighbor of the receiver is permitted to be a transmitting node in the transmission scenario, and no neighbor of the transmitter is permitted to be a receiving node in the transmission scenario. This corresponds to the assumption that a link between two cells causes such high interference at all neighboring cells of the transmitter that all links that terminate at any of these neighboring cells become inoperable, but the interference at cells that are not neighbors of the transmitter is negligible. In other words, the transmit power and cell radius are such that the interference due to a transmitting node is not felt more than one cell away. The geometry of the problem imposes a certain symmetry on all maximal transmission scenarios, such that they can be enumerated by a computer program following the above rules.

\section{Results Comparing Multihop With Single-Hop}

In this section, we solve the wireless network flow problem stated in (1-a)-(1-c) for the regular network shown in Fig. 5. The network has two full rings of EPs about the AP, and part of a third ring. The reason why only 6 out of the 18 cells in the third ring have EPs is that with 18 EPs in the third ring, the number $N$ of maximal transmission scenarios becomes too large to handle. Each throughput versus range curve has three points, corresponding to solving the wireless network flow LP problem for a network comprising: 1) only the AP and the first ring of EPs; 2) the AP and the first two rings of APs; and 3) the AP and all EPs shown in Fig. 5. The distance of the farthest EP for each of these cases yields the range of the region served by the AP by means of the multihop network of EPs. Thus, the range depends on the size of the hexagonal cells containing the EPs. The throughput versus range curves are computed for several different choices of the cell size.

1) Solution to the Single-Hop Network Flow Problem: The single-hop strategy corresponds to having transmission scenarios where there is exactly one active link in each scenario, namely with the AP as the transmitter and one EP as the receiver. Thus, there are exactly as many transmission scenarios as there are EPs, or $N=n$. If the rate from the AP $s$ to the $\mathrm{EP} i$ is $R_{s i}$ (note that there is no interference as this is the sole transmission), then the time required for EP $i$ to receive its demanded number of bits $f$ is $T_{i}=f / R_{s i}$. Since the AP transmits to each EP one by one, the total time required for all the EPs to receive their demanded number of bits is

$$
T_{1}+\cdots+T_{n}=f \sum_{i=1}^{n} \frac{1}{R_{s i}}
$$

and the throughput is, therefore

$$
\frac{f}{\sum_{i=1}^{n} T_{i}}=\frac{1}{\sum_{i=1}^{n} \frac{1}{R_{s i}}}
$$

which is the harmonic mean of the rates from the AP to the individual EPs.

2) Description of the Numerical Parameters Used in the Problem: We assume a bandwidth of $10 \mathrm{MHz}, \log$-normal shadow fading with a standard deviation of $8 \mathrm{~dB}$, and several possible choices for the radius of the hexagonal cells containing the AP and EPs. The cell containing the AP is assumed to be of the same size as the cells containing the EPs. The channel propagation model is taken to be the same Erceg-Greenstein model [20] as for the performance evaluation of the greedy transmission scenario enumeration algorithm in Section II-C, and described by the following parameters: reference distance $100 \mathrm{~m}, A=4, B=0.0065$, and $C=17.1$. In addition, we study two possible combinations of antenna transmit heights and transmit powers for the AP and the EPs, which we call Situation 1 and Situation 2, respectively.

1) Single-hop: AP transmits with $43 \mathrm{dBm}$ power with antenna height of $20 \mathrm{~m}$, and all EPs transmit with $30 \mathrm{dBm}$ power with antenna height of $10 \mathrm{~m}$.

Multihop: AP and all EPs transmit with $30 \mathrm{dBm}$ power, and AP and all EP antennas are $10 \mathrm{~m}$ high.

2) Single-hop and Multihop: AP and all EPs transmit with $30 \mathrm{dBm}$ power, and all EP antennas are $10 \mathrm{~m}$ high.

Situation 1 corresponds to the case where the AP is on a taller tower than the EPs and also can transmit with more power. This should lead to higher rates to EPs and, therefore, increase the throughput for the single-hop case over Situation 2.

3) Multihop With Omnidirectional Antennas Versus SingleHop: In Fig. 7, we plot the throughput versus range curves for several choices of the cell size for the two situations defined above. It is clear that for Situation 1, where the AP transmits with significantly higher power and from a greater height, the single-hop scheme has greater throughput for a given range compared with the multihop routing scheme. Thus, if the power available to a wired BS is large enough to cover a given area, there is no advantage to be gained in terms of throughput by introducing EPs without wired backhaul access into that area. In Situation 2, on the other hand, multihop routing is clearly superior to single-hop routing, though the advantage is not overwhelming.

4) Multihop With Narrow-Beam Antennas Versus SingleHop: We assume that, in addition to the parameters of the above model, the use of narrow-beam antennas yields a gain of $10 \mathrm{~dB}$ on each link, while contributing no interference to 


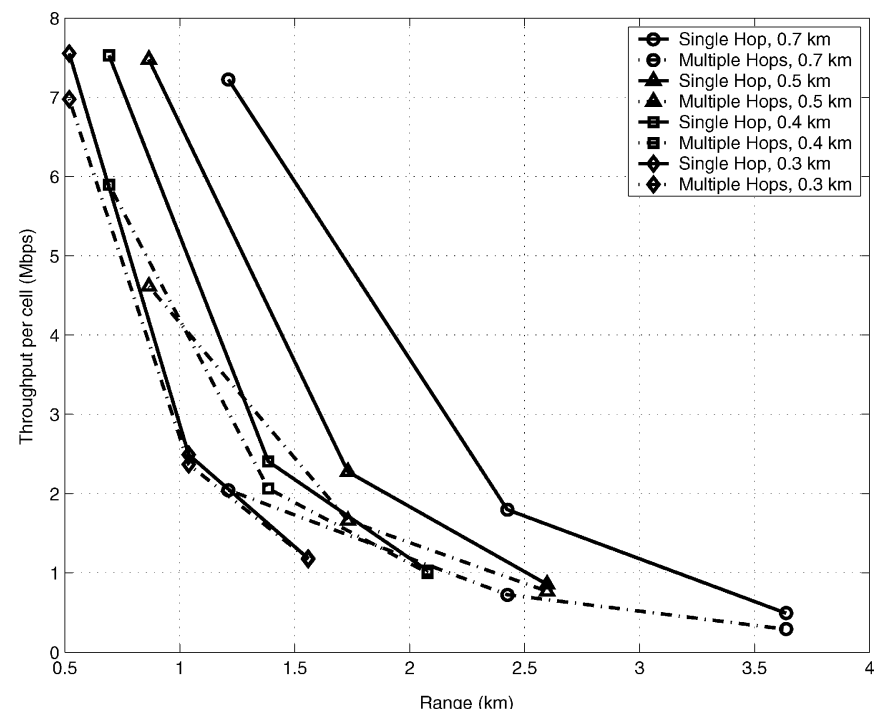

(a)

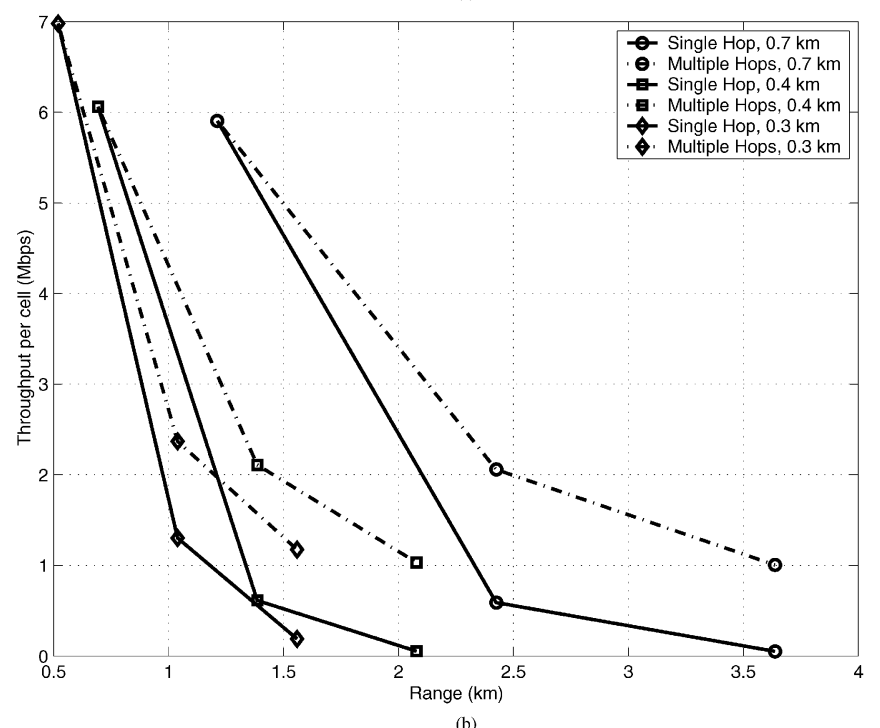

(b)

Fig. 6. Throughput versus range plots for the network in Fig. 5 for Situations 1 and 2, respectively, using narrow-beam antennas and for several choices of cell size.

any other link. In Fig. 6, we plot the throughput versus range curves for several choices of the cell size for the two Situations defined above. We observe the same advantage as for the omnidirectional antenna case for single-hop versus multihop in Situation 1, and the same advantage of multihop routing versus single-hop in Situation 2. Further, the use of narrow-beam antennas makes the gains of multihop routing over single-hop greater than if omnidirectional antennas are used.

\section{LOAD BALANCING Through Mesh BACKHAUL}

So far, we have evaluated the throughput versus range tradeoff for a multihop network with APs and EPs, concentrating on the downlink traffic. We now focus our attention on the uplink. As we discuss below, we consider a different set of performance objectives on the uplink, leading to a different problem statement. However, this new problem is still an LP and, therefore, can be solved using the techniques already developed, as detailed below.

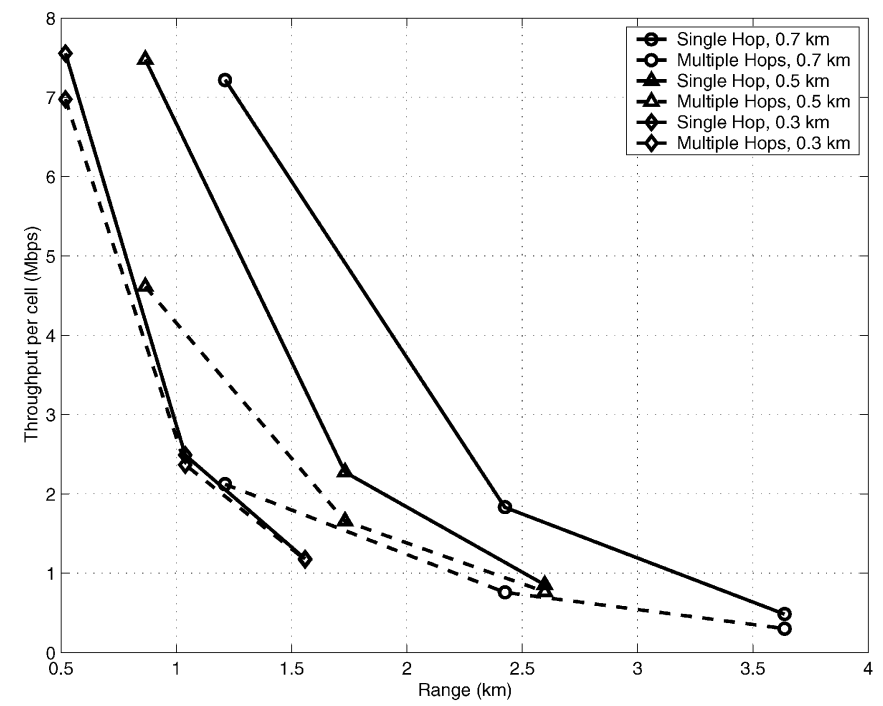

(a)

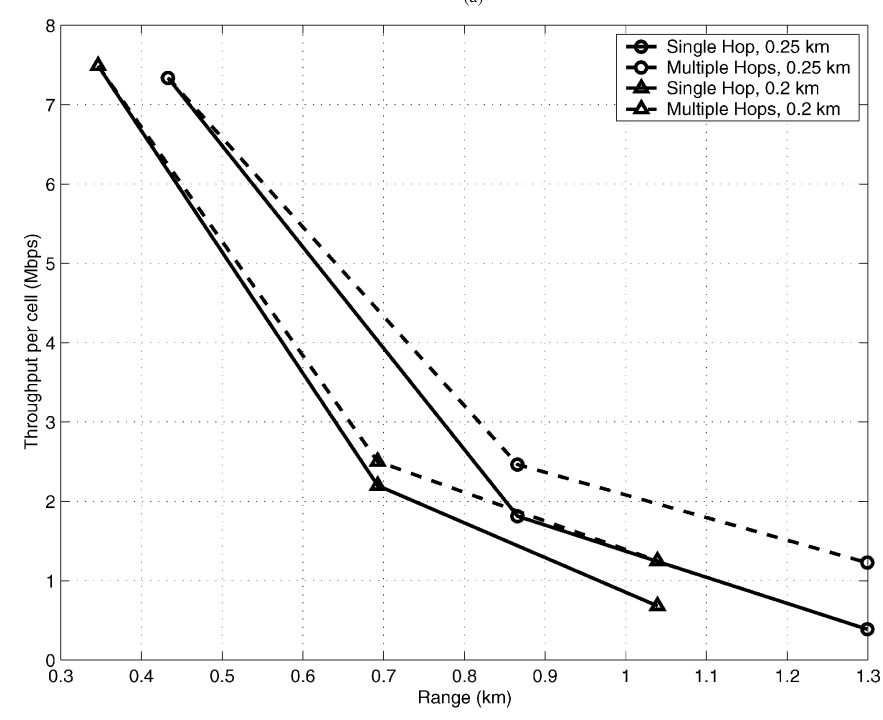

(b)

Fig. 7. Throughput versus range plots for the network in Fig. 5 for Situations 1 and 2, respectively, using omnidirectional antennas and for several choices of cell size.

\section{A. Introduction}

Consider the transmission scenario where several BSs in a wireless access network are connected to the wired network through a wired backhaul link with limited bandwidth, in addition to the wireless mesh backhaul connection between the BSs. Further suppose that the aggregate access traffic generated from end users served by each of these BSs is unequal across the network thereby creating an imbalance in the traffic that is required to be received from or transmitted back to the wired network through the wired backhaul connections. In such a system, a situation of interest could be one in which the maximum wired backhaul transmission resource is limited and some base stations' access traffic exceed the backhaul transmission rate, while other base stations' traffic is less than the backhaul transmission rate. The difference in mean traffic generated in different BSs provides an opportunity to route traffic from heavily loaded BSs to the wired network through lightly loaded BSs that do not completely consume their wired backhaul bandwidth resources. 
This can be achieved by first transmitting the traffic from the heavily loaded BS to the lightly loaded BS through multihop wireless transmission, and then forwarding to the wired network through the lightly loaded BSs wired backhaul connection. The wireless mesh backhaul allows reconfiguration of routes and traffic flow patterns to maximize the traffic carried into the wireless network. Our goal is to determine the maximum access traffic that can be carried into the wired network using the available backhaul links through the best utilization of the wireless mesh backhaul capabilities. The solution to this problem, thus, involves determining optimum routing and scheduling of flows as in Section II-B, while also taking into account the traffic generation rate and the maximum wired backhaul transmission rate from the BSs to the wired network. For a centralized routing and scheduling approach, the LP framework of the previous sections can be extended to determine the optimum routing and scheduling that maximizes the traffic carried into the wired network and this is described in the next section.

\section{B. Linear Programming Formulation}

Consider first the simpler case when there is only a single transmission scenario for all possible links with fixed link rates. All traffic generated from the different network nodes (BSs) with the wired network as the destination can be treated as a single commodity. Hence, we drop the superscript $d$ from the flow variables $x_{i j}$ defined in Section II-B. We denote the wired network node by $w$ and, hence, $x_{i w}$ represents the flow on the wired link between the node $i$ and the wired network. All flows between nodes in the network except the wired node are carried by the wireless mesh network and, thus, constrained by the transmission rate on the link. We, thus, have the following constraints:

$$
(\forall i, j) x_{i j} \leq R_{i j} T, \text { and } x_{i w} \leq R_{b} T
$$

where $R_{i j}$ is the link rate on the wireless links $(i, j), R_{b}$ is the common transmission rate of the wired links $(i, w)$, and $T$ is some fixed duration of time such as the slot time. We also have the flow conservation constraints analogous to (1-b)

$$
(\forall j) \sum_{i} x_{i j}+R_{j} T-\sum_{k} x_{j k}-x_{j w} \geq 0
$$

where $R_{j}$ is the access traffic generation rate at node $j$. The constraint is not an equality constraint because in general the access traffic generation rate can be larger than the rate at which traffic can be transmitted to other nodes over the wireless links or to the wired network over the wired link. Given the above constraints the objective is to maximize the total flow to the wired network from all the nodes, i.e.,

$$
\max \sum_{i} x_{i w}
$$

Clearly, the above optimization problem is a LP problem and can be solved using efficient methods.

The formulation above does not completely capture the effects of interference and variable-rate transmissions that are typical in wireless networks. Nevertheless, the formulation can be extended to incorporate the wireless specific aspects by identifying the set of transmission scenarios with optimum scheduling of the different transmission scenarios along the lines of Section II-B. Suppose that we have a set of $N$ transmission scenarios $\mathcal{L}_{1}, \ldots \mathcal{L}_{N}$ that operate for some proportion of times $\tau_{1}, \ldots, \tau_{N}$ of the total time $T$, where $\tau_{i} \geq 0, i=1, \ldots, N$, and $\sum_{i=1}^{N} \tau_{i}=1$. The time proportions $\tau_{i}$ are variables that are also optimized to maximize the objective. The flow variables are now specific to each transmission scenario and are denoted by $x_{i j}(t)$. The conservation constraint (5) is aggregated over all the transmission scenarios. We, thus, have the following LP problem to determine the maximum total throughput to the wired network from all the nodes in the network

$$
\max \sum_{t} \sum_{i} x_{i w}(t)
$$

subject to

$$
\begin{aligned}
& (\forall j) \quad \sum_{t} \sum_{i} x_{i j}(t)+R_{j} T \sum_{t} \tau_{t} \\
& -\sum_{t} \sum_{k} x_{j k}(t)-\sum_{t} x_{j w}(t) \geq 0 \\
& (\forall i, j) \quad x_{i j}(t) \leq R_{i j}(t) \tau_{t} T \\
& \sum_{t} \tau_{t}=1 .
\end{aligned}
$$

The total throughput is given by $\sum_{t} \sum_{i} x_{i w}(t) / T$. Without loss of generality, $T$ can be set to 1 . Note that the formulation can be easily generalized to the case of unequal transmission rates $R_{b}$ to the wired network. In the next section, we evaluate the benefit of LB using the above optimization for the regular network described in Section III-C and shown in Fig. 5.

\section{Evaluation of the Benefit of $L B$}

We consider the same regular network model illustrated in Fig. 5. However, now each of the BS-EPs is also connected to the wired network through a wired backhaul connection with maximum transmission rate of $R_{b}$. Also, we consider the reverse link or transmission of data traffic from the wireless network into the wired network. For the purposes of evaluating the benefit of LB, we assume that the access traffic generation rate falls off linearly from the center cell outwards. Fig. 8, where the proportion of the traffic generated in each BS is plotted against the location of the BS, illustrates the nonuniform traffic pattern considered. All BSs in a given hexagonal ring generate equal traffic but the traffic generation rate falls off from ring to ring going from the central cell outward. The steepness of the falloff of traffic is a parameter that determines the level of load imbalance. Since the network and the traffic pattern are homogenous in the azimuthal direction, all LB is achieved by moving traffic from the central cell outward and, thus, the same set of transmission scenarios considered previously are applicable here. The total access traffic generated from all BSs is set equal to the total backhaul bandwidth rate from all BSs to the wired network for the purpose of evaluating the benefit of LB.

Fig. 9 shows the total traffic carried back into the wired network from the wireless network with and without the multihop transmission for the omnidirectional antenna configuration. The cell radius is set at $0.5 \mathrm{~km}$ and the backhaul rate (BR) $R_{b}$ is set at 

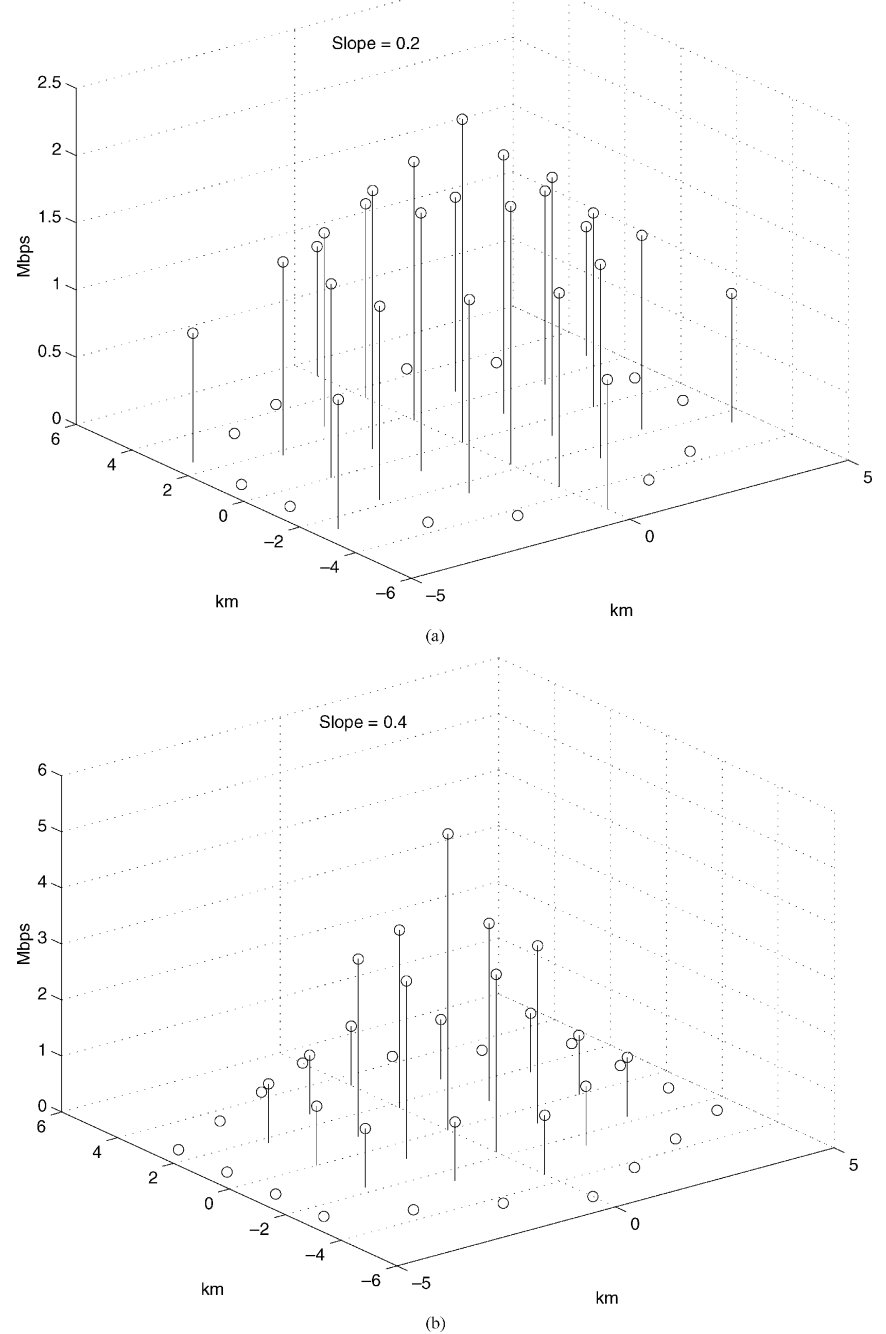

Fig. 8. Plot showing the proportion of the traffic generated in each BS versus the location of the BS for the network in Fig. 5. The traffic load decreases linearly from the central BS with slope 0.2 or 0.4 .

4 or $8 \mathrm{Mb} / \mathrm{s}$. Two traffic patterns corresponding to the slope $(S)$ parameter 0.2 and slope parameter 0.4 are considered. Clearly, LB shows a significant improvement and the larger the benefit the more unbalanced the traffic pattern. Also, for the fixed wireless bandwidth of $10 \mathrm{MHz}$, less improvement from multihop transmission is achieved as we increase the aggregate traffic rate from 4 to $8 \mathrm{Mb} / \mathrm{s}$, as expected. Similar results for the case of omnidirectional antenna configuration with cell radius of $0.1 \mathrm{~km}$ is illustrated in Fig. 10 and for the case of narrow-beam configuration with cell radius $0.5 \mathrm{~km}$ is illustrated in Fig. 11. In both of these cases, larger throughput is achieved with the multihop transmissions because of better wireless link transmission rates, thus allowing more traffic to be routed to nodes with less access traffic generation rate.

\section{SUMMARY}

A linear programming model for optimum routing and scheduling of flows in a wireless mesh network that include the effect of interference and allow for variable-rate transmissions was proposed. This framework requires the enumeration of all the

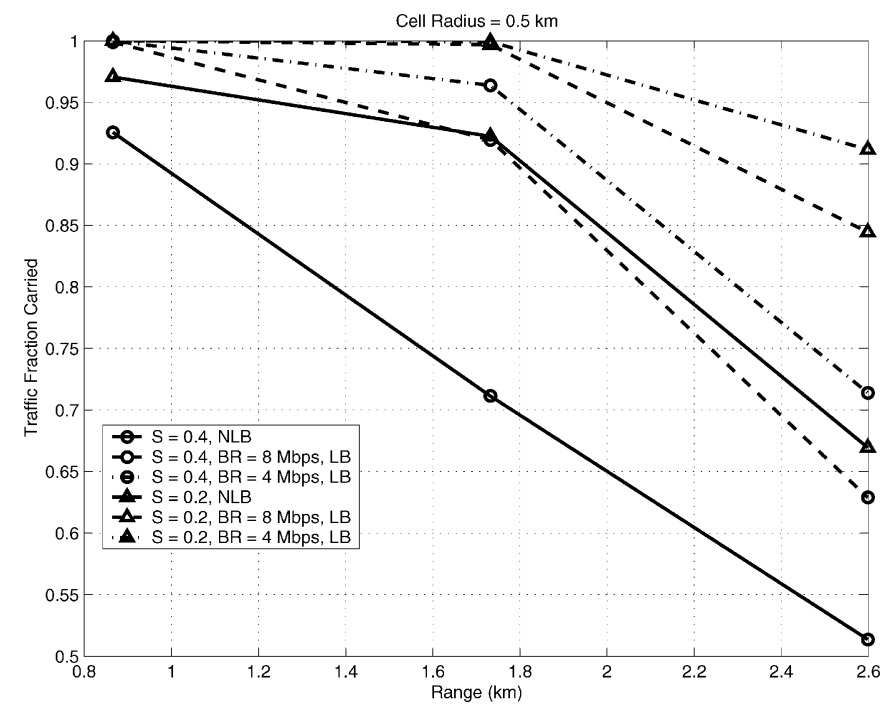

Fig. 9. The total traffic carried back into the wired network from the wireless network with and without the multihop transmission for the omnidirectional antenna configuration. The cell radius is set at $0.5 \mathrm{~km}$ and the $\mathrm{BR} R_{b}$ is set at 4 or $8 \mathrm{Mb} / \mathrm{s}$. Two traffic patterns corresponding to the slope parameter $(S)$ 0.2 and slope parameter 0.4 are considered. Clearly, LB shows a significant improvement over no load balancing (NLB) and the benefit is larger the more unbalanced the traffic pattern. Also, for the fixed wireless bandwidth of $10 \mathrm{MHz}$, less improvement from multihop transmission is achieved as we increase the aggregate traffic rate from 4 to $8 \mathrm{Mb} / \mathrm{s}$, as expected.

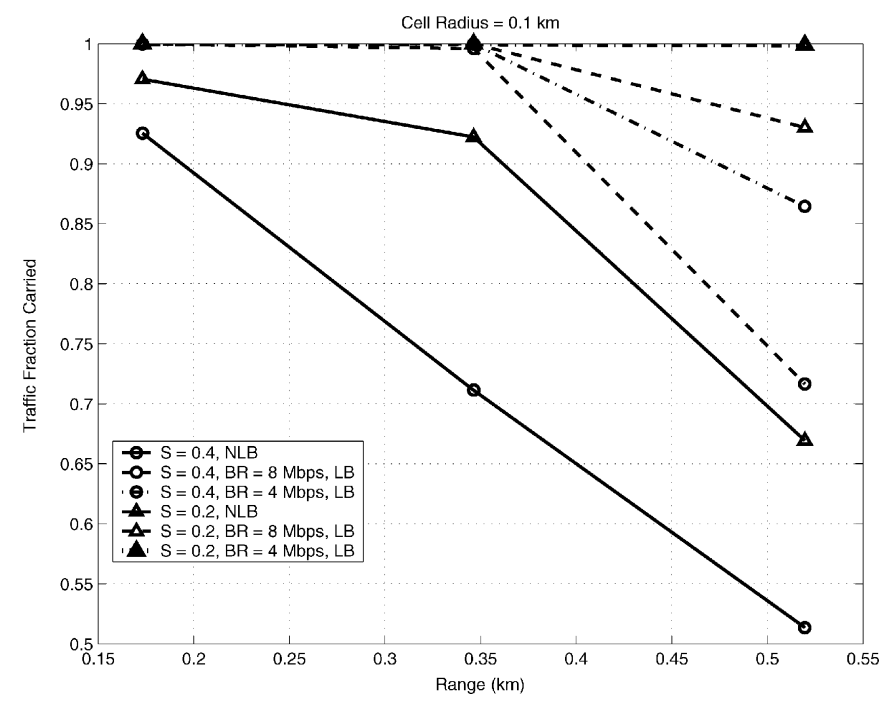

Fig. 10. The same plot as in Fig. 9, but with the cell radius set at $0.1 \mathrm{~km}$.

different transmission scenarios and the associated transmission rates. Owing to the complexity of this a heuristic algorithm was proposed for determining the important set of transmission scenarios. Simulation results showed that the proposed heuristic algorithm achieves good performance in the networks considered.

The LP framework was then applied to a regular hexagonal cellular network to evaluate the throughput-range tradeoff of wireless backhaul solutions. Throughput as a function of range was obtained for various parameter values for transmission power, antenna height, and cell radius. Results showed that the throughput per cell decays faster than linearly with the increasing number of cells served by a single wired BS. With $10 \mathrm{MHz}$ of bandwidth for backhaul, up to 18 cells arranged in 


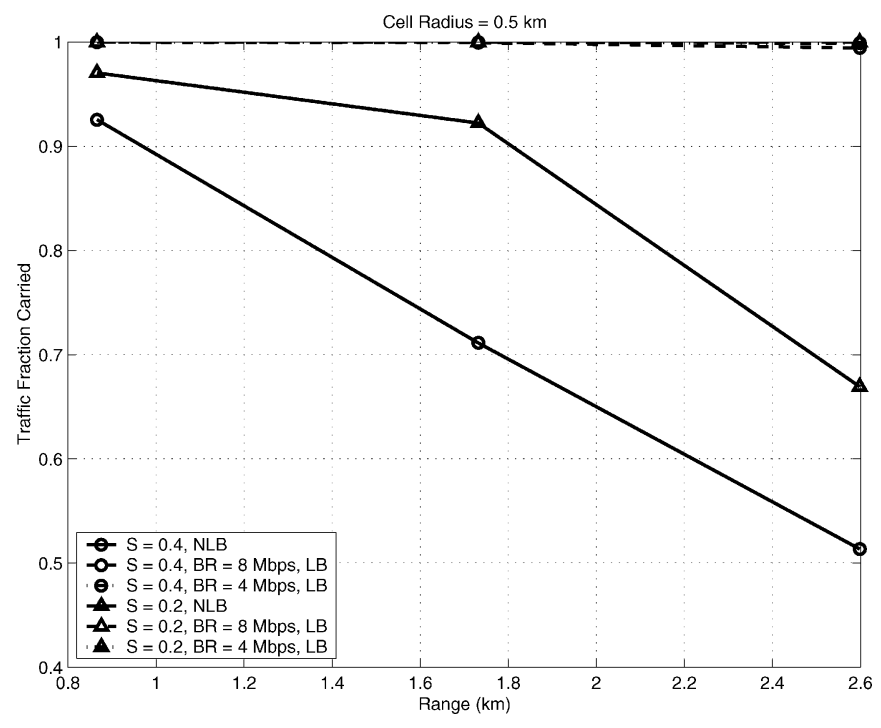

Fig. 11. The same plot as in Fig. 9, but with narrow-beam antennas instead of omnidirectional antennas.

two rings around the wired BS can be served at approximately $1.5 \mathrm{Mb} / \mathrm{s}$ per cell for a total range of about $2 \mathrm{~km}$. The sensitivity of the path loss slope parameter to the transmit antenna height results in comparable performance for single-hop transmission with $20 \mathrm{~m}$ antenna height and $43 \mathrm{dBm}$ transmit power and multihop transmission with $10 \mathrm{~m}$ antenna height and $30 \mathrm{dBm}$ transmit power. However, when the transmit powers and antenna heights are set to the same value for both single-hop and multiple-hop cases the multiple-hop outperforms the single-hop case.

The LP framework was then extended to study potential benefits of LB using the wireless mesh backhaul. The total traffic carried to the wired network is maximized by rerouting traffic to underutilized backhaul links. For transmission scenarios studied in this paper, significant gains were obtained through LB. As expected, the gains depend on the level of imbalance and the available wireless backhaul resources in comparison to the access traffic generation rate.

\section{REFERENCES}

[1] [Online]. Available: http://www.wi-fi.org/

[2] [Online]. Available: http://www.wimaxforum.org/

[3] "CDMA 2000 high-rate packet data air-interface specification," Telecommunications Industry Assoc., Arlington, VA, TIA/EIA/IS-856, 2002.

[4] "High speed downlink packet access (HSDPA), overall description," 3rd Generation Partnership Project (3GPP), TS 25.308.

[5] J. Hauser, D. Baker, and W. S. Conner. (2003) Draft project authorization request (PAR) for IEEE 802.11 extended service set (ESS) mesh. [Online]. Available: http://www.ieee802.org/11/PARs/11-04-0054-020mes-par-ieee-802-11-ess-mesh.doc

[6] IEEE 802.16-2004, "IEEE Standard for local and metropolitan area networks Part 16: Air interface for fixed broadband wireless access systems," IEEE Prod. SH95246-TBR., 2004.

[7] B. H. Walke, R. Pabst, D. Schultz, P. Herhold, H. Yanikomeroglu, S. Mukherjee, H. Viswanathan, M. Lott, W. Zirwas, M. Dohler, H. Aghvami, D. D. Falconer, and G. P. Fettweis, "Relay-based deployment concepts for wireless and mobile broadband radio," IEEE Commun. Mag., vol. 42, no. 9, pp. 80-89, Sep. 2004.
[8] H. Viswanathan and S. Mukherjee, "Performance of cellular networks with relays and centralized scheduling," IEEE Trans. Wireless Commun., vol. 4, no. 5, pp. 2318-2328, Sep. 2005.

[9] J. Cho and Z. J. Haas, "On the throughput enhancement of the downstream channel in cellular radio networks through multihop relaying," IEEE J. Sel. Areas Commun., vol. 22, no. 7, pp. 1206-1219, Sep. 2004.

[10] S. Toumpis and A. J. Goldsmith, "Capacity regions for wireless ad hoc networks," IEEE Trans. Wireless Commun., vol. 2, no. 4, pp. 736-748, Jul. 2003.

[11] H. Luo, R. Ramjee, P. Sinha, L. Li, and S. Lu, "UCAN: A unified cellular and ad-hoc network architecture," in Proc. MobiCom, San Diego, CA, Sep. 2003, pp. 353-367.

[12] H. Wu, C. Qiao, S. De, and O. Tonguz, "Integrated cellular and ad hoc relaying systems: iCAR," IEEE J. Sel. Areas Commun., vol. 19, no. 10, pp. 2105-2115, Oct. 2001.

[13] A. J. Viterbi, CDMA: Principles of Spread Spectrum Communication. Reading, MA: Addison-Wesley, 1995.

[14] R. L. Cruz and A. V. Santhanam, "Optimal routing, link scheduling and power control in multi-hop wireless networks," in Proc. INFOCOM, Mar./Apr. 2003, pp. 702-711.

[15] M. Kodialam and T. Nandagopal, "Characterizing achievable rates in multi-hop wireless networks: The joint routing and scheduling problem," in Proc. MobiCom, San Diego, CA, Sep. 2003, pp. 42-54.

[16] T. ElBatt and A. Ephremides, "Joint scheduling and power control for wireless ad hoc networks," IEEE Trans. Wireless Commun., vol. 3, no. 1, pp. 74-85, Jan. 2004.

[17] C. M. Barnhart, J. E. Wieselthier, and A. Ephremides, "A neural network approach to solving the link activation problem in multihop radio networks," IEEE Trans. Commun., vol. 43, no. 2/3/4, pp. 1277-1283, Feb./Mar./Apr. 1995.

[18] T. M. Cover and J. A. Thomas, Elements of Information Theory. New York: Wiley, 1991.

[19] K. Kumaran and L. Qian, "Uplink scheduling in CDMA packet-data systems," in Proc. INFOCOM, Mar./Apr. 2003, pp. 292-300.

[20] V. Erceg, L. J. Greenstein, S. Y. Tjandra, S. R. Parkoff, A. Gupta, B. Kulic, A. A. Julius, and R. Bianchi, "An empirically based path loss model for wireless channels in suburban environments," IEEE J. Sel. Areas Commun., vol. 17, no. 7, pp. 1205-1211, Jul. 1999.

[21] V. Chvátal, Linear Programming. San Francisco, CA: Freeman, 1983.

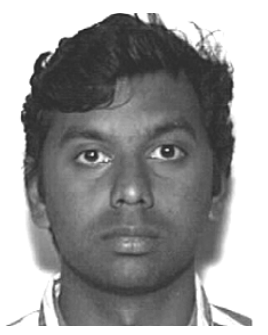

Harish Viswanathan (SM'03) received the B. Tech. degree from the Department of Electrical Engineering, Indian Institute of Technology, Madras, in 1992, and the M.S. and Ph.D. degrees from the School of Electrical Engineering, Cornell University, Ithaca, NY, in 1995 and 1997, respectively.

$\mathrm{He}$ is presently with Bell Laboratories, Lucent Technologies, Murray Hill, NJ. His research interests include information theory, communication theory, wireless networks, and signal processing.

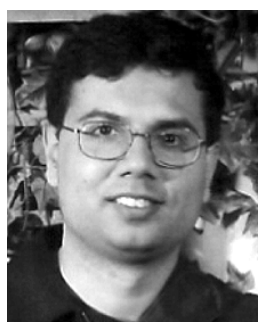

Sayandev Mukherjee (M'92-SM'05) was born in Bangalore, India, in 1970 . He received the B. Tech. degree in electrical engineering from the Indian Institute of Technology, Kanpur, in 1991, and the M.S. and Ph.D. degrees in electrical engineering from Cornell University, Ithaca, NY, in 1994 and 1997, respectively.

Since 1996, he has been a Member of Technical Staff in the Wireless Research Laboratory, Bell Laboratories, Lucent Technologies, Murray Hill, NJ. His research interests include stochastic models, wireless system simulations, and connectivity issues in ad hoc wireless networks. 